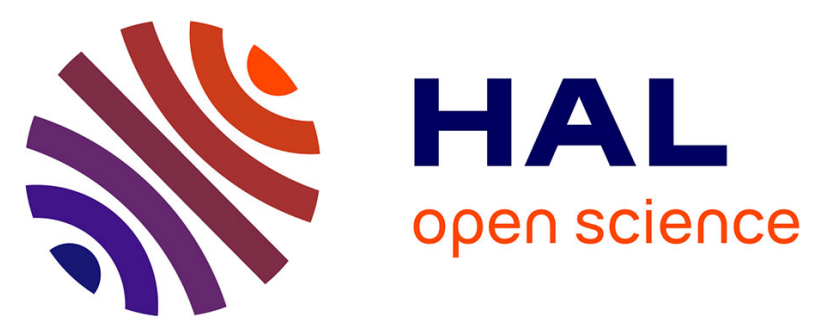

\title{
Decreased expression of EZH2 is associated with upregulation of ER and favorable outcome to tamoxifen in advanced breast cancer
}

E. A. Reijm, M. P. H. M. Jansen, K. Ruigrok-Ritstier, I. L. Staveren, M. P. Look, M. E. Meijer Gelder, A. M. Sieuwerts, S. Sleijfer, J. A. Foekens, E. M. J. J. Berns

\section{To cite this version:}

E. A. Reijm, M. P. H. M. Jansen, K. Ruigrok-Ritstier, I. L. Staveren, M. P. Look, et al.. Decreased expression of EZH2 is associated with upregulation of ER and favorable outcome to tamoxifen in advanced breast cancer. Breast Cancer Research and Treatment, 2010, 125 (2), pp.387-394. 10.1007/s10549-010-0836-9 . hal-00555000

\section{HAL Id: hal-00555000 https://hal.science/hal-00555000}

Submitted on 12 Jan 2011

HAL is a multi-disciplinary open access archive for the deposit and dissemination of scientific research documents, whether they are published or not. The documents may come from teaching and research institutions in France or abroad, or from public or private research centers.
L'archive ouverte pluridisciplinaire HAL, est destinée au dépôt et à la diffusion de documents scientifiques de niveau recherche, publiés ou non, émanant des établissements d'enseignement et de recherche français ou étrangers, des laboratoires publics ou privés. 
Decreased expression of EZH2 is associated with upregulation of ER and favorable outcome to tamoxifen in advanced breast cancer

E.A. Reijm”, M.P.H.M. Jansen ", K. Ruigrok-Ritstier, I.L. van Staveren, M.P. Look, M.E. Meijervan Gelder, A.M. Sieuwerts, S. Sleijfer, J.A. Foekens, E.M.J.J. Berns

Department of Medical Oncology, Erasmus MC/Daniel den Hoed Cancer Center/Josephine Nefkens Institute, Rotterdam, The Netherlands

Keywords: EZH2, ESR1, Tamoxifen, siRNAs, advanced disease

*E.A. Reijm and M.P.H.M. Jansen contributed equally.

Acknowledgement of research support: This study was supported in part by TI Pharma, Leiden, The Netherlands, project no. T3-108.

Correspondence to: M.P.H.M. Jansen, PhD, Erasmus MC, Department of Medical Oncology, Josephine Nefkens Institute, Room Be401, P.O.Box 2040, 3000 CA Rotterdam, The Netherlands.

Phone: +31107044373; Fax: +31107044377; e-mail: m.p.h.m.jansen@erasmusmc.nl 


\section{Abstract}

Purpose: To investigate EZH2 in a large series of breast cancer patients for its prognostic and predictive value, and to evaluate its functional role in treatment response in vitro.

Experimental design: EZH2 levels were measured using quantitative Real-Time Polymerase Chain Reaction (qRT-PCR) in primary breast cancer specimens and related to clinicopathologic factors and disease outcome. EZH2 expression was downregulated with siRNAs in MCF7, to assess expression alterations of putative EZH2 downstream genes and to determine cell numbers after treatment with the anti-estrogen ICI 164.384.

Results: In 688 lymph node-negative patients who did not receive adjuvant systemic therapy, EZH2 was not significantly correlated with metastasis-free survival (MFS). In 278 patients with advanced disease treated with first-line tamoxifen monotherapy, the tertile with highest EZH2 levels was associated with the lowest clinical benefit $(\mathrm{OR}=0.48 ; \mathrm{P}=0.02)$ and with a shorter progression-free survival (PFS) in both univariate $(H R=1.80 ; P<0.001)$ and multivariate analysis, including traditional factors $(\mathrm{HR}=1.61 ; \mathrm{P}=0.004)$.

In vitro, EZH2 silencing in MCF7 caused a 38\% decrease in cell numbers $(P<0.001)$ whereas ICI 164.384 treatment resulted in a $25 \%$ decrease $(\mathrm{P}<0.001)$ compared to controls. Combining EZH2 silencing with $\mathrm{ICl}$-treatment reduced cell numbers with $67 \%(\mathrm{P}<0.001)$ compared to control conditions. EZH2 downregulation was associated with an almost 2-fold upregulation of the estrogen receptor alpha $(E R)(P=0.001)$.

Conclusion: $\mathrm{EZH} 2$ has no prognostic value in breast cancer. High levels of EZH2 are associated with poor outcome to tamoxifen therapy in advanced breast cancer. Downregulated EZH2 leads to upregulation of the ER and better response to anti-estrogens. 


\section{Introduction}

The anti-estrogen tamoxifen has been used for treatment of estrogen receptor alpha (ER) positive breast cancer for more than 20 years both in the adjuvant and advanced setting. Although the majority of breast tumors express the ER, approximately half of the patients with ER-positive advanced disease does not respond to endocrine therapy or will eventually develop resistance. As a consequence, there is a high need for markers to identify patients likely to benefit from tamoxifen and to get a better insight into mechanisms conferring resistance.

In a previous genome-wide profiling study in breast cancer patients with advanced disease, we revealed an 81-gene signature for resistance to first-line tamoxifen treatment [1]. One of the gene families from this profile is Enhancer of Zeste, consisting of Enhancer of Zeste Homolog 1 (EZH1, OM IM 601674) and Enhancer of Zeste Homolog 2 (EZH2, OMIM 601573). Both EZ homologs belong to Polycomb group (PcG) proteins, and are involved in transcriptional control and epigenetic memory maintenance for preservation of cellular characteristics [2]. EZH2 comprises the core of the Polycomb Repressor Complex 2 (PRC2) [3-5], has histone lysine methyltransferase activity, and mediates di- and trimethylation on histone 3 lysine residue 27 (H3K27) [2]. EZH1 can also be a part of PRC2, although with low histone lysine methyltransferase activity [6]. We previously explored EZH1 with qRT-PCR, but did not observe a significant correlation with clinical outcome in 229 ER-positive tumors of patients with advanced disease treated with first-line tamoxifen monotherapy [7].

In contrast to EZH1, EZH2 has been extensively studied in malignancies. Increased expression of $\mathrm{EZH} 2$ in breast, prostate and bladder cancer have been associated with a high histological grade and increased tumor cell proliferation [8-10]. In addition, EZH2 was identified as an adverse prognostic marker for breast and prostate cancer, but these studies included only small series of patients [11,12]. Its predictive value for outcome to tamoxifen in advanced breast cancer has, however, not been studied yet.

The aims of the current study were (1) to assess the prognostic value of EZH2 in a large series of patients, (2) to study its predictive value for outcome to tamoxifen treatment in advanced breast cancer and (3) to explore its functional role in endocrine therapy resistance. 


\section{Patients and methods}

Patients

This retrospective study, in which coded tumor tissues were used, has been approved by the medical ethics committee of the Erasmus MC Rotterdam, The Netherlands (MEC 02.953), was performed in accordance with the Code of Conduct of the Federation of Medical Scientific Societies in the Netherlands (http://www. fmwv.nl), and reported following the REMARK recommendations [13], wherever possible. Frozen breast tumor tissue specimens of female patients with primary operable breast cancer who entered the clinic between 1979 and 1996 were analyzed. Follow-up, tumor staging, and response to therapy was defined by standard International Union Against Cancer (Geneva, Switzerland) classification criteria [14]. Tumor protein expression levels of ER and progesterone receptor $(\mathrm{PgR})$ were determined [15], and $10 \mathrm{fmol} / \mathrm{mg}$ cytosolic protein was used as cut-off point to classify tumors as ER and/or PgR-positive. The following criteria were applied to include tumor specimens from final analysis: (1) sufficient frozen tumor material, (2) more than $30 \%$ epithelial tumor cells in haematoxylin and eosin stained sections, (3) breast tumor tissue specimen of good RNA quality according to predefined criteria [16]; and (4) EZH2 mRNA expression levels were measured and reference mRNA levels were detectable. After applying the inclusion criteria, tumor specimens and clinical data of 1,318 patients were available for analysis. From these 1,318 patients (for clinicopathologic details, see Supplementary Table S1) 580 patients (44\%) underwent breast conserving lumpectomy and 738 patients modified mastectomy (56\%). The median follow-up time of patients alive was 90 months, range 4-231 months. Eight hundred eighty-nine patients did not receive adjuvant systemic therapy, while 429 patients (33\%; all lymph node-positive) did; 198 (15\%) were treated with hormonal therapy, 216 (16\%) with chemotherapy (70 patients anthracycline-based (FAC/FEC) and 146 patients nonanthracycline-based (CMF)) and 15 patients received both hormonal and chemotherapy.

\section{Hormonal therapy of advanced disease}

ER-positive tumors of 249 patients (out of the 1,318 M0 patients) who developed advanced disease after treatment for primary breast cancer and who received first-line tamoxifen therapy were included in this study. This set was completed with 29 tumors of patients with 
distant metastases at initial diagnosis (M1 patients). These 278 patients were divided according to response to tamoxifen. Clinical benefit from tamoxifen, defined as a complete or partial response according to standard International Union Against Cancer (Geneva, Switzerland) classification criteria [14] or no change longer than 6 months after treatment initiation (stable disease), was observed in 173 patients (62\%); 11 patients showed complete response, 38 a partial response and 124 patients had stable disease. No clinical benefit occurred in 105 patients (38\%).

\section{$R N A$ isolation and quantitative real-time $P C R$}

Tissue processing, RNA isolation, cDNA synthesis, quantitative real-time polymerase chain reaction ( $\mathrm{qRT}-\mathrm{PCR}$ ) and expression data generation were performed as described previously [16]. The qRT-PCR assays were carried out on an ABI Prism 7700 Sequence Detection System (Applied Biosystems, Nieuwerkerk a/d IJssel, The Netherlands) or a MX3000P Realtime PCR system (Stratagene, Amsterdam, The Netherlands). Assay-on-Demand kits (Applied Biosystems) were used to measure mRNA levels of EZH2 (Hs00544830_m1) and ER (Hs00174860_m1; measures ESR1). Primer sequences of the reference genes PGBD, HPRT, B2M have been described [16]. Forty rounds of amplification were performed and fluorescent signals of Taqman probes were used to generate Cycle threshold $(\mathrm{Ct})$ values to calculate mRNA expression levels. Expression levels of EZH2 and ER were normalized against average expression levels of three reference genes in tumor samples and against HPRT levels in cell lines [16].

\section{Breast cancer cell line and RNA interference}

MCF7, an estrogen sensitive ER-positive breast cancer cell line, was cultured in RPMI 1640 containing phenol red and 10\% heat-inactivated fetal calf serum (Sigma-Aldrich Chemie). To perform EZH2 knockdown experiments, small interfering RNA (siRNA) targeting EZH2 mRNA (Qiagen, Venlo, The Netherlands) was used according recommendations and described previously [17]. Two different siRNA duplexes for EZH2 were pooled with target sequences: r(CCAUGUUUACAACUAUCAA)dTdT (sense) and r(UUGAUAGUUGUAAACAUGG)dTdT (antisense) for the first duplex and r(GCAAAUUCUCGGUGUCAAA)dTdT (sense) and 
r(UUUGACACCGAGAAUUUGC)dTdT (antisense) for the second duplex. As control, MCF7 cells were transfected with non-specific silencing pool of siRNAs (Qiagen). siRNAs (5nM) were introduced via inverse transfection into MCF7, using HiPerfect transfection reagent (Qiagen). Six experiments were independently performed at different time points in $24(\mathrm{~N}=1)$ or 96 wells plates $(\mathrm{N}=5)$. The 24 and 96 wells plate contained 330,000 cells and 6,000 cells per well, respectively, and 2 and 8 wells per condition was used and pooled for further analyses. Within three experiments in 96 wells plates, part of the cells was harvested after 96 hours for mRNA and protein analysis. The remaining part was again transfected with siRNAs, and subsequently grown for 96 hours in standard culture medium supplemented with the pure ER antagonist ICI $164.384(100 \mathrm{nM})$ or with ethanol vehicle alone as control. The pure ER antagonist ICI was used to exclude the agonistic effects of tamoxifen. To assess the effect of EZH2 silencing, cell numbers were counted with a coulter counter at day 4 and 8 . To determine response to 96 hours of ICl164.384 treatment cell numbers were counted at day 8 . Throughout an experiment, culture medium was renewed every 3 days and at the end cells were lysed and RNA and protein isolated using the MirVana Paris kit (Ambion, Foster City, CA, USA).

\section{Immunocytochemistry and Western blotting}

Cytospins were prepared from MCF7 cells of above experiments, fixed with $1 \%$ formaldehy de and incubated with a monoclonal antibody against EZH2 (BD Biosciences, San Jose, CA, $(1: 2000))$ and a secondary peroxidase conjugated rabbit anti-mouse antibody. EZH2 protein expression was visualized with a diaminobenzidine staining reaction. Western blotting of protein samples were performed as described previously [17]. Antibodies against EZH2 (monoclonal (1:2000), BD Biosciences, 1:2000), H3K27 (polyclonal (1:5000), ABCAM, Cambridge, MA, USA) and GAPDH (monoclonal (1:500), Chemicon Inc., Temecula, CA, USA) were used and detected with horseradish peroxidase (HRP)-conjugated or HRPpolymer (DAKO Real Envision, DAKO, Diagnostica $\mathrm{GmbH}$, Hamburg, Germany) labeled secondary antibodies and chemiluminescent reagents (ECL-kit, Pierce, Rockford, IL). The Scanalytics One-D program (Alpha Innotech Ltd., Cannock, UK) was used for quantification. 
Data analysis and statistics

The relationship of EZH2 expression levels with patient and tumor characteristics was investigated using nonparametric methods, i.e., Spearman rank correlations for continuous variables and Wilcoxon rank-sum or Kruskal-Wallis test for ordered variables. For the analyses with continuous variables, mRNA levels of EZH2 were log transformed and of ER and $\mathrm{PgR}$ were box-cox transformed to reduce skewness of the distribution. Logistic regression analysis was used to calculate the odds ratio $(\mathrm{OR})$ that defines the relation between expression levels and clinical benefit from therapy. The Cox proprotional hazards model was used to compute the hazard ratio $(\mathrm{HR})$ in the analysis of metastasis -free survival (MFS), overall survival (OS) and progression-free survival (PFS). MFS and OS were previously described [18]. PFS was defined as the time elapsed between initiation of tamoxifen therapy and the first detection of disease progression. In multivariate analysis, logistic and Cox regression analysis was applied to determine whether EZH2 had predictive value and was independent when added to the base model of traditional factors. The Cox proportional hazard assumption was not violated as verified by a test based on Schoenfeld residuals. Both HR and OR were represented with their 95\% confidence intervals $(95 \% \mathrm{Cl})$. Survival curves were generated using the Kaplan and Meier method and a log rank test was used to test for differences. Computations were done with the STATA statistical package, release 10.1 (STATA Corp., College Station, TX). In the in vitro studies, a student t-test was performed to test for significance for differences in cell counts and RNA levels. All P-values were two-sided and $\mathrm{P}<0.05$ was considered statistically significant. 


\section{Results}

Associations of EZH2 mRNA expression levels with clinicopathological factors

In this study, we determined the mRNA expression levels in 1,318 primary breast carcinomas. Median expression levels of EZH2, its interquartile range, and its association with patient and tumor characteristics are summarized in Supplementary Table S1. Briefly, high EZH2 mRNA levels were significantly associated with younger age, premenopausal status, poor histologic grade, larger tumor size, and status of ER, PgR, ERBB2 and EGFR. An inverse correlation between $E Z H 2$ and $E R$ was observed $\left(P<0.001, r_{s}=-0.33\right)$. Expression of $E Z H 2$ was higher in ERBB2 positive samples compared with ERBB2 negative samples $(P<0.001)$.

\section{EZH2 levels and clinical outcome}

The prognostic value of EZH2 was assessed in 688 lymph node-negative (LNN) patients who did not receive any adjuvant systemic therapy. EZH2 levels, as continuous variable, were not significantly correlated with metastasis-free survival (MFS) (HR=1.14, $95 \% \mathrm{Cl}$ : $0.98-1.32$; $\mathrm{P}=0.10)$. Considering overall survival $(\mathrm{OS}), \mathrm{EZH} 2$ was significantly associated in $\mathrm{LNN}$ patients (HR=1.25, 95\% Cl: 1.07-1.46; $P=0.006)$.

\section{Association of EZH2 levels with clinical benefit and progression-free survival} In univariate analysis, increasing EZH2 expression levels as continuous variable were significantly associated with a lower clinical benefit in patients with advanced breast cancer treated with first-line tamoxifen monotherapy $(n=278)(\mathrm{OR}=0.68,95 \% \mathrm{Cl}: 0.47-0.98 ; \mathrm{P}=0.04)$ (Supplementary Table S2). In analogy, after categorizing EZH2 expression levels into tertiles, the highest tertile was significantly associated with a lower clinical benefit to tamoxifen therapy $(\mathrm{OR}=0.48,95 \% \mathrm{Cl}: 0.26-0.89 ; \mathrm{P}=0.02)$. In multivariate analysis, however, when corrected for traditional predictive factors including age, menopausal status, DSR, DFS, ER and $\mathrm{PgR}$ levels, no significant association with clinical benefit was observed (Supplementary Table S2). In univariate analysis, increasing EZH2 expression levels analyzed as continuous variable were significantly associated with shorter PFS (HR=1.28, 95\% Cl: 1.08-1.51; $\mathrm{P}=0.004$ ) (Table 1). In univariate analysis, compared with tumors in the lowest tertile of $E Z \mathrm{H} 2$ expression, those with the highest EZH2 levels were associated with a poor PFS (HR=1.80, 
95\% Cl: 1.32-2.46; $\mathrm{P}<0.001$ ) (Table 1, Figure 1). Remarkably, the intermediate and highest expression groups have a similar PFS during the first 12 months while the curves diverge thereafter. In multivariate analysis, compared with tumors with EZH2 levels in the lowest tertile, those with highest EZH2 levels were significantly associated with poor PFS (HR=1.61, 95\% Cl: 1.16-2.24; $P=0.004)$. Moreover, ER levels were not significantly $(P=0.21)$ different between the 3 different tertiles of EZH2 (Supplemental Figure S1).

\section{EZH2 and RNA interference}

To investigate how $E Z H 2$ is functionally involved in response to anti-estrogens, we performed in vitro studies in the ER-positive human breast cancer cell line MCF7. To this end MCF7 cells were treated with ICl164.384 combined with non-silencing (NS) or EZH2 silencing. When cells transfected with siNS were exposed to $100 \mathrm{nM}$ ICI164.384 a decrease in cell numbers of $25 \%$ (range $12-30 \%, N=3, P<0.001$ ) after 96 hours was observed, confirming that MCF7 is an anti-estrogen sensitive cell line. Knockdown experiments showed an average silencing level of $\mathrm{EZH} 2$ of $79 \%$ (range $64 \%-93 \%, \mathrm{~N}=6, \mathrm{P}<0.001$ ) after 96 hours (Figure 2). EZH2 silencing caused a significant decrease in cell numbers of $38 \%$ (range $17-53 \%, \mathrm{~N}=3$, $\mathrm{P}<0.001$ ) compared with controls (Figure 3 ). When EZH2 silencing and ICl-treatment were combined, cell numbers were reduced with $67 \%$ (range $54-75 \%, N=3, P<0.001$ ) compared to non-silenced MCF7 cells. Both immunocytochemistry of cytospins and Western blotting confirm knockdown of EZH2 on the protein level after 96 hours of EZH2 silencing (Figure 4). To validate EZH2 silencing functionally we demonstrated (Figure 4) that methylation of lysine residue 27 of histone 3 diminishes when silencing EZH2.

\section{Association between EZH2 levels and ER expression}

In view of the inverse correlation between EZH2 and ER mRNA expression levels observed in our 1,318 breast cancer tumor specimens, we next studied the effect of EZH2 silencing on ER expression in vitro. Downregulation of EZH2 in MCF7 cells (79\% after 96 hours, range 64\%$93 \%, \mathrm{~N}=6)$ was associated with an average two-fold upregulation of $\mathrm{ER}(\mathrm{N}=6$, range 1.5-2.3, $\mathrm{P}=0.001$ ) (Figure 2), which is concordant with the observed inverse correlation between $E Z \mathrm{H} 2$ and ER mRNA expression in clinical samples. 


\section{Discussion}

We demonstrate here for the first time that a significant association exists between high levels of EZH2 with outcome in terms of PFS for ER-positive breast cancer patients treated with first-line tamoxifen for advanced disease. Additionally, it was shown that EZH2 expression impacts response to tamoxifen rather than reflecting tumor aggressiveness since no prognostic value for EZH2 was revealed in LNN primary breast cancer who did not receive adjuvant systemic therapy.

In contrast to our findings, EZH2 has previously been suggested to bear prognostic value in breast cancer. Based on in silico analysis, on a relatively small set of 78 tumors of young LNN patients ( $<55$ years) with low grade tumors [19], Kleer et al. reported that mRNA expression of EZH2 was significantly higher in invasive carcinomas that metastasize within 5 years of primary diagnosis compared with invasive carcinomas that did not [11]. In another study immunohistochemistry was used to demonstrate an association between EZH2 expression and increased tumor cell proliferation in melanoma, prostate, endometrial, and breast cancer [9]. Association of high EZH2 expression with unfavorable prognosis was revealed in all investigated tumor types with the exception of breast cancer as survival data were not available. We observed that EZH2 associates significantly with overall survival, however, overall survival is not only dependent on tumor aggressiveness but also on treatment and response of the patient in the adjuvant and/or advanced disease setting. That EZH2 in our study predicts poor overall survival in LNN patients who did not obtain adjuvant systemic therapy suggests an association with treatment response in the advanced disease setting, as for example shown for first-line tamoxifen monotherapy in this study. Collett et al. also used immunohistochemistry to assess the prognostic value of EZH2 in breast cancer [8]. In 190 tumors (100 EZH2-negative and 90 EZH2-positive), they demonstrated a significant correlation between EZH2 positivity and high disease stage at time of diagnosis in terms of locally advanced disease or metastatic disease. However, this group comprises only 6 patients with metastatic disease at initial diagnosis. Additionally, this set was heterogeneous with both LNN and LN-positive tumors. And though treatment status was not discussed, it is reasonable to assume that LNP patients have received systemic treatment thereby impacting outcome and obscuring EZH2's true prognostic value. However, in our study population 
comprising the largest series of patients studied so far, 688 LNN patients, who did not receive any adjuvant systemic therapy enabling to assess the true prognostic value of EZH2, we could not confirm its prognostic value. This discrepancy in findings remains to be clarified but might be due to the methodology applied and the cohorts of patients studied.

Furthermore, we found an association between EZH2 and the type of response to therapy in advanced disease with the highest EZH2 levels related to poor outcome. In order to elucidate the potential underlying mechanisms for this association, we explored the association between EZH2 expression and ER expression. In our 1,318 breast cancer tumor specimens, we observed an inverse association between EZH2 and ER mRNA expression levels. Accordingly, in silico analysis of 15 independent breast cancer datasets of the Oncomine database (supplementary Figure S2), with in total 2,437 samples (713 ER-negative, 1724 ERpositive), confirmed this finding. In addition, we performed functional studies in which EZH2 expression was silenced with siRNAs in the human estrogen sensitive breast cancer cell line MCF7. The decrease in cell numbers following downregulating EZH2 expression in MCF7 suggests that $\mathrm{EZH} 2$ has an effect on cell proliferation, in agreement with previously performed studies showing an association between EZH2 and cell proliferation $[8,11]$. We have shown that growth inhibition in the $\mathrm{ICl}+\mathrm{siEZH} 2$ experiment $(67 \%$ inhibition) adds up the effect of EZH2 silencing and ICl164,384 treatment on MCF7 growth (38\% and $25 \%$ inhibition, respectively). These combined $\mathrm{ICl}+\mathrm{siEZH} 2$ experiments demonstrate no overlap in growth inhibition and indicates that the effect of EZH2 silencing on MCF7 growth is independent of the effect of ER inhibition by $\mathrm{ICl}$. Our observed upregulation of ER by silencing of EZH2 and the inverse correlation between EZH2 and ER status (ER-negative vs ER-positive) in breast cancer specimens (Supplemental Figure S2), however, suggest an EZH2 and ER interaction which may also result in enhanced sensitivity to ICl164.384. Although the observed two-fold upregulation of ER does not seem impressive at first glance, it is already observed after 96 hours of culturing. Moreover, MCF7 is a cell line with already one of the highest ER expression levels in our panel of 39 breast cancer cell lines (data not shown). In that perspective, an almost 2-fold upregulation can be regarded substantial. Furthermore, the extent of ER upregulation by silencing EZH2 is in the range of what has been described for other downstream factors. For example, Yu et al published a 1.6-fold upregulation of the 
Adrenergic Receptor when silencing EZH2 [20]. We hypothesize that the promoter region of ER lacks DNA methylation in MCF7 and that the observed upregulation of ER is predominantly due to decreased histone H3K27 trimethylation caused by EZH2 knockdown. Further studies are needed to verify this hypothesis.

Given the presumed role of EZH2 in the regulation of ER expression, EZH2 might be an interesting target for therapy. Recently, Varambally et al. discovered a physiological EZH2inhibitor, miRNA-101, which inhibits the expression and function of EZH2 in cancer cell lines [21]. It has been shown that miRNA-101 expression diminishes during cancer progression, resulting in an increased EZH2 expression and concomitant dysregulation of epigenetic pathways. This cascade is thought to underlie progression of several types of cancers, e.g., prostate, brain and lung cancer [21]. In addition, also miRNA-26a has been reported to posttranscriptionally repress EZH2 [22]. Recently, Kota et al. demonstrated the capacity of miRNA-26a as an anti-tumor therapy in a mouse model of hepatocellular carcinomas, where it resulted in inhibition of cancer cell proliferation and induction of tumor-specific apoptosis [23]. As a result, it would be interesting to further investigate $\mathrm{EZH} 2$ as a potential target for therapy, and include miRNA-101 and miRNA-26a as 'in vivo' inhibitors.

In conclusion, EZH2 has no prognostic value in our large set of LNN adjuvant untreated breast cancer patients. However, high EZH2 levels are associated with unfavorable outcome to tamoxifen treatment in breast cancer patients with advanced disease, which suggests that EZH2 can be used as a predictive marker. Moreover, downregulation of EZH2 caused additional growth inhibition next to anti-estrogen therapy in vitro and resulted in ER upregulation. If validated, EZH2 may be considered to serve as a potential target to increase the anti-tumor activity of anti-estrogen therapies in breast cancer. Furthermore, its assessment may contribute to a more appropriate selection of patients for tamoxifen therapy and thereby a more tailored management of patients with breast cancer.

\section{Disclosure of potential conflicts of interest}

No potential conflicts of interest were disclosed. 


\section{References}

1. Jansen MP, Foekens JA, van Staveren IL et al (2005) Molecular classification of tamoxifen-resistant breast carcinomas by gene expression profiling. J Clin Oncol 23:732-740.

2. Schuettengruber B, Chourrout D, Vervoort M et al (2007) Genome regulation by polycomb and trithorax proteins. Cell 128:735-745.

3. Cao R, Wang L, Wang $\mathrm{H}$ et al (2002) Role of histone H3 lysine 27 methylation in Polycomb-group silencing. Science 298:1039-1043.

4. Czermin B, Melfi R, McCabe D et al (2002) Drosophila enhancer of Zeste/ESC complexes have a histone $\mathrm{H} 3$ methyltransferase activity that marks chromosomal Polycomb sites. Cell 111:185-196.

5. Kuzmichev A, Nishioka K, Erdjument-Bromage $\mathrm{H}$ et al (2002) Histone methyltransferase activity associated with a human multiprotein complex containing the Enhancer of Zeste protein. Genes Dev 16:2893-2905.

6. Margueron R, Li G, Sarma K et al (2008) Ezh1 and Ezh2 maintain repressive chromatin through different mechanisms. Mol Cell 32:503-518.

7. Jansen M, Foekens J, Ritstier K et al (2005) A miniPathway for tamoxifen therapy resistance. Breast Cancer Res and Treat 94:S31-S31.

8. Collett K, Eide GE, Arnes $\mathrm{J}$ et al (2006) Expression of enhancer of zeste homologue 2 is significantly associated with increased tumor cell proliferation and is a marker of aggressive breast cancer. Clin Cancer Res 12:1168-1174.

9. Bachmann IM, Halvorsen OJ, Collett $\mathrm{K}$ et al (2006) EZH2 expression is associated with high proliferation rate and aggressive tumor subgroups in cutaneous melanoma and cancers of the endometrium, prostate, and breast. J Clin Oncol 24:268-273.

10. Raman JD, Mongan NP, Tickoo SK et al (2005) Increased expression of the polycomb group gene, EZH2, in transitional cell carcinoma of the bladder. Clin Cancer Res 11:8570-8576.

11. Kleer CG, Cao Q, Varambally $S$ et al (2003) EZH2 is a marker of aggressive breast cancer and promotes neoplastic transformation of breast epithelial cells. Proc Natl Acad Sci USA 100:11606-11611. 
12. Varambally S, Dhanasekaran SM, Zhou M et al (2002) The polycomb group protein EZH2 is involved in progression of prostate cancer. Nature 419:624-629.

13. McShane LM, Altman DG, Sauerbrei W, et al (2006) REporting recommendations for tumor MARKer prognostic studies (REMARK). Breast Cancer Res and Treat 100:229-235.

14. Hayward JL, Carbone PP, Heuson JC et al (1977) Assessment of response to therapy in advanced breast cancer: a project of the Programme on Clinical Oncology of the International Union Against Cancer, Geneva, Switzerland. Cancer 39:12891294.

15. Foekens JA, Portengen H, van Putten WL et al (1989) Prognostic value of estrogen and progesterone receptors measured by enzyme immunoassays in human breast tumor cytosols. Cancer Res 49:5823-5828.

16. Sieuwerts AM, Meijer-van Gelder ME, Timmermans M et al (2005) How ADAM-9 and ADAM-11 differentially from estrogen receptor predict response to tamoxifen treatment in patients with recurrent breast cancer: a retrospective study. Clin Cancer Res 11:7311-7321.

17. Jansen MP, Ruigrok-Ritstier K, Dorssers LC et al (2009) Downregulation of SIAH2, an ubiquitin E3 ligase, is associated with resistance to endocrine therapy in breast cancer. Breast Cancer Res and Treat 116:263-271.

18. van Agthoven T, Sieuwerts AM, Veldscholte $\mathrm{J}$ et al (2009) CITED2 and NCOR2 in anti-oestrogen resistance and progression of breast cancer. $\mathrm{Br} \mathrm{J}$ Cancer 101:18241832.

19. van 't Veer LJ, Dai H, van de Vijver MJ et al (2002) Gene expression profiling predicts clinical outcome of breast cancer. Nature 415:530-536.

20. Yu J, Cao Q, Mehra $R$ et al (2007) Integrative genomics analysis reveals silencing of beta-adrenergic signaling by polycomb in prostate cancer. Cancer Cell 12:419-431.

21. Varambally S, Cao Q, Mani RS et al (2008) Genomic loss of microRNA-101 leads to overexpression of histone methyltransferase EZH2 in cancer. Science 322:16951699.

22. Wong CF, Tellam RL (2008) MicroRNA-26a targets the histone methyltransferase 
Enhancer of Zeste homolog 2 during myogenesis. J Biol Chem 283:9836-9843.

23. Kota J, Chivukula RR, O'Donnell KA et al (2009) Therapeutic microRNA delivery suppresses tumorigenesis in a murine liver cancer model. Cell 137:1005-1017. 


\section{Legends}

Table 1: Cox uni- and multivariate analysis for progression-free survival of EZH2, continuous and categorized, in estrogen receptor-positive tumors from 278 patients whose recurrence was treated with first-line tamoxifen monotherapy.

Figure 1: Kaplan-Meier curves of progression-free survival (PFS) as a function of EZH2 mRNA expression levels. Patients were evenly divided into three groups according to EZH2 mRNA levels. Patients at risk at different timepoints are indicated.

Figure 2: EZH2 and ER mRNA expression levels after EZH2 silencing (siEZH2) in MCF7 cells. Average level of siEZH2 was $79 \%$ after 96 hours of culturing. Downregulation of EZH2 was associated with an average upregulation of ER of almost two-fold.

Bars represent mean $\pm \mathrm{sd}$ of triplicate measurements expressed as fold-difference compared with non-silenced MCF7 cells. Data shown are of six time-point independent cell culture experiments.

Figure 3: EZH2 silencing (siEZH2) in MCF7 and response to ICI 164,384 treatment. MCF7 cells were cultured for 8 days in RPMI containing 10\% FCS and transfected with siRNAs at day 0 and again at day 4. Culture medium was supplemented with ICI 164,384 after 96 hours. Cell numbers were measured in siEZH2 and/or ICI 164,384 at day 8.

Bars represent mean $\pm \mathrm{sd}$ of triplicate measurements expressed as fold-difference compared with untreated and non-silenced MCF7 cells. Results were obtained from 3 time-point independent cell culture experiments.

Figure 4: Immunocytochemistry of cytospins and western blotting. EZH2 protein expression after 96 hours EZH2 silencing (siEZH2) compared with non-silenced MCF7 cells (siNS) are shown by immunocytochemistry (top). Western blotting (bottom) shows histone 3 lysine 27 trimethylation (H3K27me3). 
Supplementary Table S1. Association of EZH2 mRNA expression levels with patient and tumor characteristics.

Supplementary Table S2: Logistic uni- and multivariate regression analysis for clinical benefit of EZH2, continuous and categorized, in estrogen receptor-positive tumors from 278 patients whose recurrence was treated with first-line tamoxifen therapy.

Supplemental Figure S1: Evaluation of ER levels in the 3 tertiles of EZH2, illustrated in a boxplot, in 235 ER-positive tumors of patients with advanced disease treated with first-line tamoxifen. A Kruskal-Wallis rank test showed that $E R$ levels were not significantly $(P=0.21)$ different between the 3 tertiles of EZH2.

Supplementary Figure S2: In silico analysis of 15 public datasets available in ONCOMINE (www.oncomine.org) showed higher EZH2 mRNA expression levels in ER-negative breast tumors (blue bars) compared to ER-positive breast tumors (red bars). 
Table 1: Cox uni- and multivariate analysis for progression-free survival of EZH2, continuous and categorized, in estrogen receptor-positive tumors from 278 patients whose recurrence was treated with first-line tamoxifen monotherapy.

\begin{tabular}{|c|c|c|c|c|c|c|c|}
\hline \multirow[t]{2}{*}{ Factor of base model } & & \multicolumn{3}{|c|}{ Univariate analysis } & \multicolumn{3}{|c|}{ Multivariate analysis* } \\
\hline & & $\mathrm{HR}$ & $95 \% \mathrm{CI}$ & $\mathrm{P}$ & $\mathrm{HR}$ & $95 \% \mathrm{CI}$ & $\mathbf{P}$ \\
\hline \multicolumn{8}{|l|}{ Age $(y)$} \\
\hline$\leq 55$ & $104(37.4 \%)$ & 1.00 & & & 1.00 & & \\
\hline $56-70$ & $102(36.7 \%)$ & 0.83 & $0.62-1.10$ & 0.19 & 0.68 & $0.45-1.03$ & 0.07 \\
\hline$>70$ & 72 (25.9\%) & 0.68 & $0.50-0.94$ & 0.02 & 0.60 & $0.38-0.93$ & 0.02 \\
\hline \multicolumn{8}{|l|}{ Menopausal status } \\
\hline Premenopausal & $68(24.5 \%)$ & 1.00 & & & & & \\
\hline Postmenopausal & $210(75.5 \%)$ & 0.90 & $0.67-1.19$ & 0.45 & & & \\
\hline \multicolumn{8}{|l|}{ Disease-free survival } \\
\hline$\leq 1$ year & $72(25.9 \%)$ & 1.00 & & & 1.00 & & \\
\hline $1-3$ years & $124(44.6 \%)$ & 0.69 & $0.51-0.92$ & 0.01 & 0.67 & $0.49-0.90$ & 0.009 \\
\hline$>3$ years & $82(29.5 \%)$ & 0.51 & $0.36-0.71$ & $<0.001$ & 0.50 & $0.36-0.71$ & $<0.001$ \\
\hline \multicolumn{8}{|l|}{ Dominant site of relapse } \\
\hline Soft tissue & $29(10.4 \%)$ & 1.00 & & & 1.00 & & \\
\hline Bone & $145(52.2 \%)$ & 1.29 & $0.85-1.96$ & 0.24 & 1.29 & $0.81-2.01$ & 0.26 \\
\hline Viscera & $104(37.4 \%)$ & 1.18 & $0.76-1.83$ & 0.45 & 1.39 & $0.87-2.21$ & 0.17 \\
\hline ER mRNA & $278(100 \%)$ & 0.90 & $0.86-0.96$ & $<0.001$ & 0.91 & $0.86-0.97$ & 0.004 \\
\hline PgR mRNA & $278(100 \%)$ & 0.90 & $0.85-0.96$ & 0.001 & 0.92 & $0.86-0.98$ & 0.01 \\
\hline \multicolumn{5}{|l|}{ Factors analyzed } & \multicolumn{3}{|c|}{ Additions to base model } \\
\hline \multicolumn{8}{|l|}{ EZH2 mRNA } \\
\hline \multicolumn{8}{|l|}{ Categorized variable } \\
\hline $\mathrm{t} 1$ & $93(33.5 \%)$ & 1.00 & & & 1.00 & & \\
\hline t2 & $93(33.5 \%)$ & 1.40 & $1.04-1.89$ & 0.03 & 1.48 & $1.08-2.02$ & 0.02 \\
\hline t3 & $92(33.0 \%)$ & 1.80 & $1.32-2.46$ & $<0.001$ & 1.61 & $1.16-2.24$ & 0.004 \\
\hline
\end{tabular}


Figure 1: Kaplan-Meier curves of progression-free survival (PFS) as a function of EZH2 mRNA expression levels. Patients were evenly divided into three groups according to EZH2 mRNA levels. Patients at risk at different timepoints are indicated.

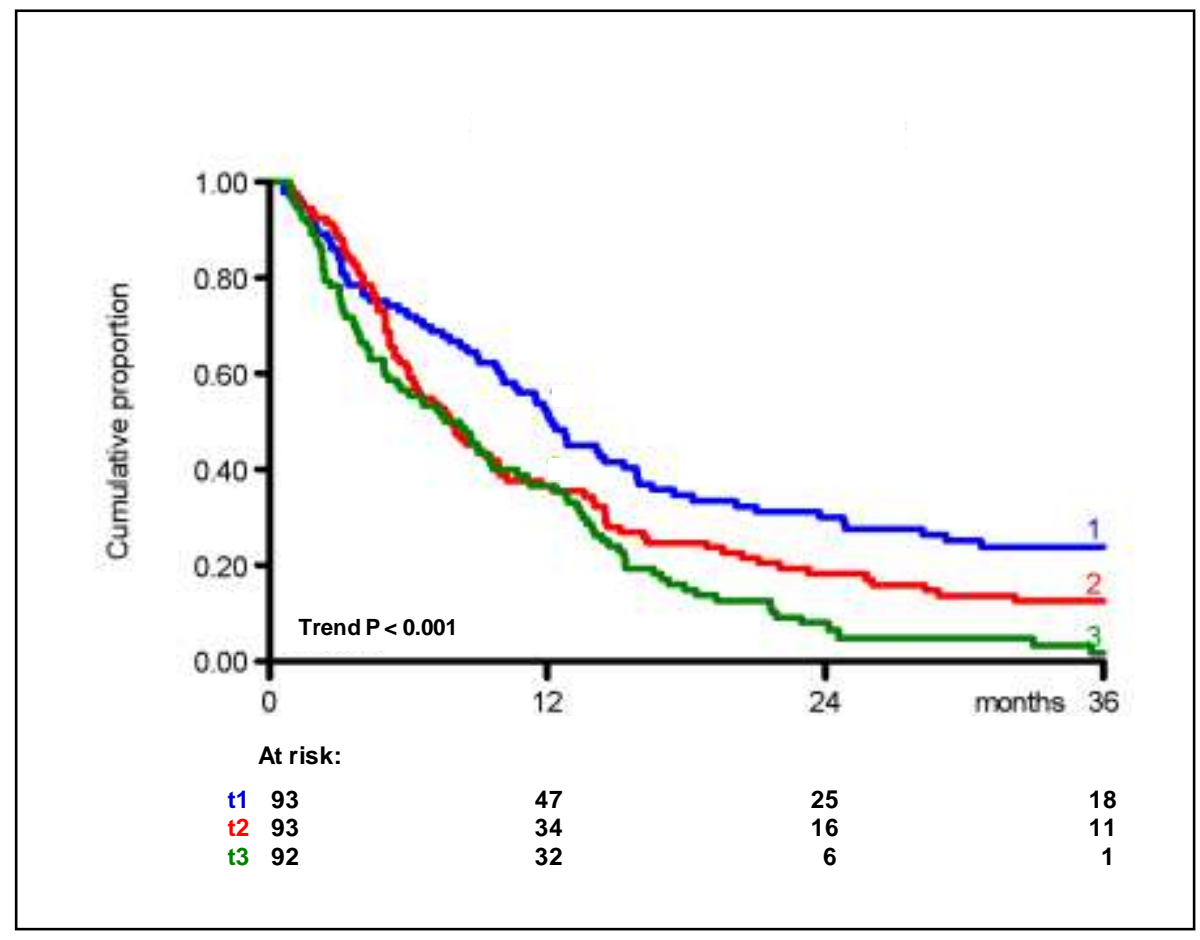


Figure 2: EZH2 and ER mRNA expression levels after EZH2 silencing (siEZH2) in MCF7 cells. Average level of siEZH2 was $79 \%$ after 96 hours of culturing. Downregulation of EZH2 was associated with an average upregulation of ER of almost two-fold.

Bars represent mean $\pm s d$ of triplicate measurements expressed as fold-difference compared with non-silenced MCF7 cells. Data shown are of six time-point independent cell culture experiments.

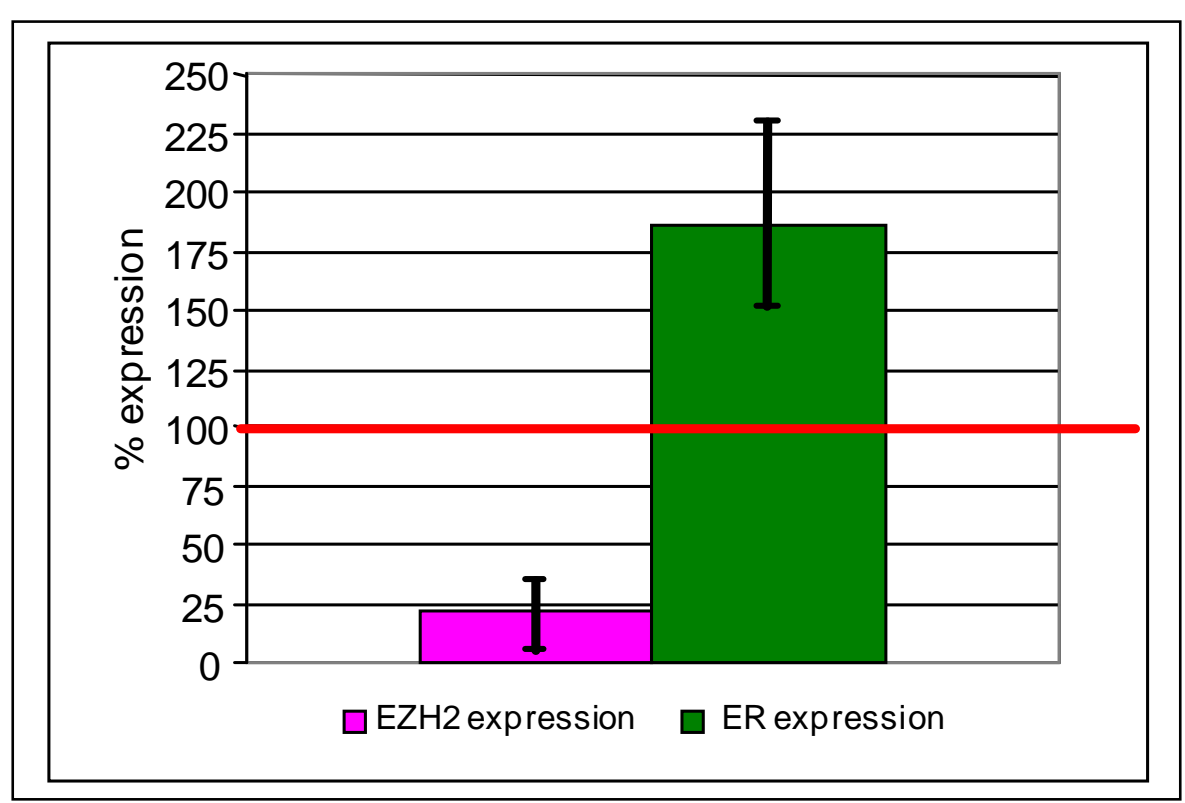


Figure 3: EZH2 silencing (siEZH2) in MCF7 and response to ICI 164,384 treatment. MCF7 cells were cultured for 8 days in RPMI containing 10\% FCS and transfected with siRNAs at day 0 and again at day 4. Culture medium was supplemented with ICI 164,384 after 96 hours. Cell numbers were measured in siEZH2 and/or ICI 164,384 at day 8.

Bars represent mean $\pm \mathrm{sd}$ of triplicate measurements expressed as fold-difference compared with untreated and non-silenced MCF7 cells. Results were obtained from 3 time-point independent cell culture experiments.

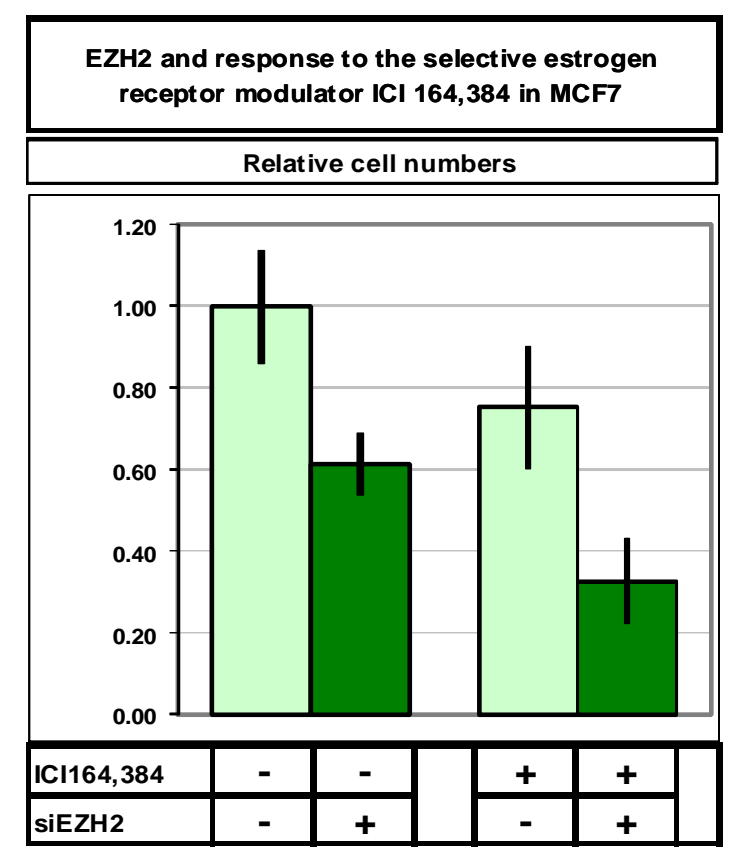


Figure 4: Immunocytochemistry of cytospins and western blotting. EZH2 protein expression after 96 hours EZH2 silencing (siEZH2) compared with non-silenced MCF7 cells (siNS) are shown by immunocytochemistry (top). Western blotting (bottom) shows histone 3 lysine 27 trimethylation (H3K27me3).

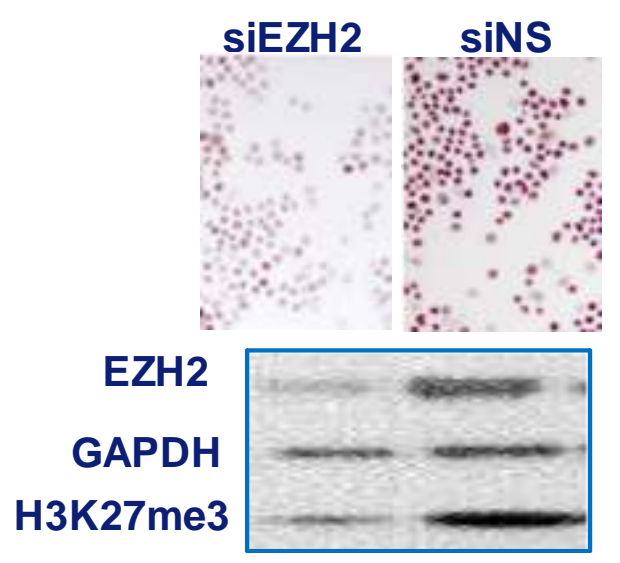


Supplementary Table S1. Association of EZH2 mRNA expression levels with patient and tumor characteristics.

\begin{tabular}{|c|c|c|c|c|}
\hline & \multirow[b]{3}{*}{$1318(100 \%)$} & \multicolumn{3}{|c|}{ EZH2 mRNA levels } \\
\hline & & Median & iqr & $\mathbf{P}$ \\
\hline \multirow{2}{*}{\multicolumn{4}{|c|}{ Age $(y)$}} & \multirow{6}{*}{$<0.001^{\#}$} \\
\hline & & & & \\
\hline$\leq 40$ & $163(12.4 \%)$ & 0.13 & 0.14 & \\
\hline $41-55$ & $493(37.4 \%)$ & 0.12 & 0.12 & \\
\hline $56-70$ & $443(33.6 \%)$ & 0.11 & 0.11 & \\
\hline$>70$ & $219(16.6 \%)$ & 0.10 & 0.10 & \\
\hline \multicolumn{2}{|l|}{ Menopausal status } & & & \multirow[t]{3}{*}{$0.019^{\dagger}$} \\
\hline Premenopausal & $547(41.5 \%)$ & 0.12 & 0.12 & \\
\hline Postmenopausal & $771(58.5 \%)$ & 0.11 & 0.11 & \\
\hline \multicolumn{2}{|l|}{ Histologic grade ${ }^{\ddagger}$} & & & \multirow[t]{3}{*}{$<0.001^{*}$} \\
\hline Poor & $734(55.7 \%)$ & 0.13 & 0.12 & \\
\hline Good/moderate & $187(14.2 \%)$ & 0.09 & 0.10 & \\
\hline \multicolumn{2}{|l|}{ Tumor size } & & & \multirow[t]{4}{*}{$0.008^{*}$} \\
\hline $\mathrm{pT} 1, \leq 2 \mathrm{~cm}$ & $417(31.6 \%)$ & 0.11 & 0.10 & \\
\hline pT $2,2-5 \mathrm{~cm}$ & $749(56.8 \%)$ & 0.12 & 0.12 & \\
\hline $\mathrm{pT} 3,>5 \mathrm{~cm}$ and $\mathrm{pT} 4$ & $152(11.5 \%)$ & 0.12 & 0.10 & \\
\hline \multicolumn{2}{|l|}{ Lymph nodes involved } & & & \multirow[t]{4}{*}{$0.338^{*}$} \\
\hline 0 & $688(52.2 \%)$ & 0.11 & 0.12 & \\
\hline $1-3$ & $281(21.3 \%)$ & 0.11 & 0.11 & \\
\hline$>3$ & $349(26.5 \%)$ & 0.13 & 0.10 & \\
\hline \multicolumn{2}{|l|}{$\mathrm{ER} \alpha$ protein status $\ddagger$} & & & \multirow[t]{3}{*}{$<0.001^{\#}$} \\
\hline Negative & $350(26.7 \%)$ & 0.17 & 0.16 & \\
\hline Positive & $962(73.3 \%)$ & 0.10 & 0.09 & \\
\hline \multicolumn{2}{|l|}{ PgR protein status ${ }^{\ddagger}$} & & & \multirow[t]{3}{*}{$<0.001^{\#}$} \\
\hline Negative & $449(36.0 \%)$ & 0.16 & 0.16 & \\
\hline Positive & $797(64.0 \%)$ & 0.10 & 0.08 & \\
\hline \multicolumn{2}{|l|}{ ERBB2 status $\ddagger$} & & & \multirow[t]{3}{*}{$<0.001^{\dagger}$} \\
\hline Negative & $1080(83.0 \%)$ & 0.11 & 0.10 & \\
\hline Positive & $222(17.0 \%)$ & 0.16 & 0.13 & \\
\hline \multicolumn{2}{|l|}{ EGFR expression ${ }^{\ddagger}$} & & & \multirow[t]{3}{*}{$<0.001^{\#}$} \\
\hline Below median & $652(50.0 \%)$ & 0.10 & 0.11 & \\
\hline Above median & $651(50.0 \%)$ & 0.14 & 0.14 & \\
\hline \multicolumn{5}{|c|}{$\begin{array}{l}\text { Abbreviation: iqr, interquartile range (q75-q25). } \\
{ }^{*} \text { Kruskal-Wallis test. } \\
\text { † Wilcoxon rank-sum (Mann-Whitney) Utest. } \\
\text { † Unknown: Grade, } n=397(30.1 \%) ; \text { ER } \alpha \text { status, } n=6(0.5 \%) ; \text { PgR status, } n=72(5.5 \%) ; \text { ERBB2 } \\
\quad \text { (cutpoint }=18.01355), n=16(1.2 \%) ; E G F R, n=15(1.1 \%) . \\
\text { \# Spearman rank correlation. }\end{array}$} \\
\hline
\end{tabular}


Supplementary Table S2: Logistic uni- and multivariate regression analysis for clinical benefit of EZH2, continuous and categorized, in estrogen receptor-positive tumors from 278 patients whose recurrence was treated with first-line tamoxifen therapy.

\begin{tabular}{|c|c|c|c|c|c|c|c|}
\hline \multirow[t]{2}{*}{ Factor of base model } & & \multicolumn{3}{|c|}{ Univariate analysis } & \multicolumn{3}{|c|}{ Multivariate analysis } \\
\hline & & OR & $95 \% \mathrm{CI}$ & $P$ & OR & $95 \% \mathrm{CI}$ & $P$ \\
\hline \multicolumn{8}{|l|}{ Age $(y)$} \\
\hline$\leq 55$ & $104(37.4 \%)$ & 1.00 & & & 1.00 & & \\
\hline $56-70$ & $102(36.7 \%)$ & 1.52 & $0.86-2.67$ & 0.15 & 1.06 & $0.45-2.47$ & 0.90 \\
\hline$>70$ & $72(25.9 \%)$ & 1.59 & $0.85-2.96$ & 0.15 & 1.16 & $0.47-2.85$ & 0.74 \\
\hline \multicolumn{8}{|l|}{ Menopausal status } \\
\hline Premenopausal & $68(24.5 \%)$ & 1.00 & & & 1.00 & & \\
\hline Postmenopausal & $210(75.5 \%)$ & 1.54 & $0.88-2.68$ & 0.13 & 1.17 & $0.49-2.80$ & 0.72 \\
\hline \multicolumn{8}{|l|}{ Disease-free survival } \\
\hline$\leq 1$ year & $72(25.9 \%)$ & 1.00 & & & 1.00 & & \\
\hline $1-3$ years & $124(44.6 \%)$ & 3.11 & $1.70-5.69$ & $<0.001$ & 3.28 & $1.74-6.18$ & $<0.001$ \\
\hline$>3$ years & $82(29.5 \%)$ & 4.04 & 2.05-7.97 & $<0.001$ & 4.22 & $2.06-8.68$ & $<0.001$ \\
\hline \multicolumn{8}{|l|}{ Dominant site of relapse } \\
\hline Soft tissue & $29(10.4 \%)$ & 1.00 & & & 1.00 & & \\
\hline Bone & $145(52.2 \%)$ & 0.64 & $0.27-1.50$ & 0.30 & 0.57 & $0.22-1.45$ & 0.24 \\
\hline Viscera & $104(37.4 \%)$ & 0.85 & $0.35-2.06$ & 0.72 & 0.61 & $0.23-1.63$ & 0.33 \\
\hline ER mRNA & $278(100 \%)$ & 1.20 & $1.07-1.34$ & 0.001 & 1.19 & $1.05-1.36$ & 0.006 \\
\hline PgR mRNA & $278(100 \%)$ & 1.12 & $0.98-1.27$ & 0.09 & 1.06 & $0.92-1.22$ & 0.43 \\
\hline \multicolumn{5}{|l|}{ Factors analyzed } & \multicolumn{3}{|c|}{ Additions to base model } \\
\hline \multicolumn{8}{|l|}{ EZH2 mRNA } \\
\hline Continuous variable & $278(100 \%)$ & 0.68 & $0.47-0.98$ & 0.04 & 0.74 & $0.49-1.12$ & 0.16 \\
\hline \multicolumn{8}{|l|}{ Categorized variable } \\
\hline $\mathrm{t} 1$ & $93(33.5 \%)$ & 1.00 & & & 1.00 & & \\
\hline t2 & $93(33.5 \%)$ & 0.56 & $0.30-1.04$ & 0.07 & 0.53 & $0.27-1.03$ & 0.06 \\
\hline $\mathrm{t} 3$ & $92(33.0 \%)$ & 0.48 & $0.26-0.89$ & 0.02 & 0.6 & $0.30-1.20$ & 0.15 \\
\hline
\end{tabular}


Supplemental Figure S1: Evaluation of ER levels in the 3 tertiles of EZH2, illustrated in a boxplot, in 235 ER-positive tumors of patients with advanced disease treated with first-line tamoxifen. A Kruskal-Wallis rank test showed that $E R$ levels were not significantly $(P=0.21)$ different between the 3 tertiles of EZH2.

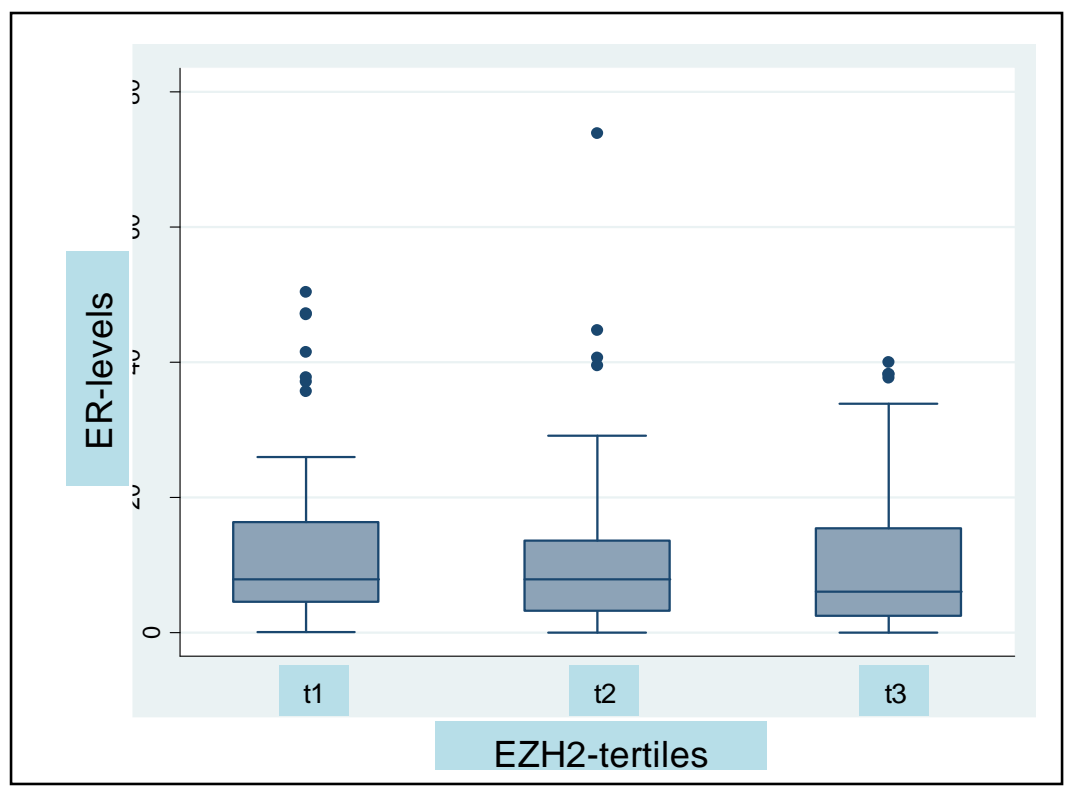


Supplemental Figure S2: In silico analysis of 15 public datasets available in ONCOMINE (www.oncomine.org) showed higher EZH2 mRNA expression levels in ER-negative breast tumors (blue bars) compared to ER-positive breast tumors (red bars).

Analys is of public datas ets available in Oncomine (March 2009): EZH2 expression levels and ER status in Breast Cancer

\begin{tabular}{|c|c|c|c|c|c|c|}
\hline Analys is & Author & Paper & \# pts & ERneg & ERpos & P-value \\
\hline 1 & Desmedt & Clin Cancer Res 2007 & $198 \mathrm{pts}$ & 64 & 134 & $3,90 \mathrm{E}-14$ \\
\hline 2 & Bittner & http://expo.intgen.org/geo/is tPublic GeoTransactions.do & $336 \mathrm{pts}$ & 78 & 154 & $6,40 \mathrm{E}-12$ \\
\hline 3 & Wang & Lancet 2005 & $286 \mathrm{pts}$ & 77 & 209 & $7,00 E-10$ \\
\hline 4 & vandeVijver & N Engl J Med 2002 & $295 \mathrm{pts}$ & 69 & 226 & $3,20 \mathrm{E}-07$ \\
\hline 5 & Miller & PNAS 2005 & $251 \mathrm{pts}$ & 34 & 213 & $1,20 \mathrm{E}-06$ \\
\hline 6 & Saal & PNAS 2007 & $105 \mathrm{pts}$ & 60 & 45 & $3,00 E-06$ \\
\hline 7 & Ivshina (subset of Miller-p53 set) & Cancer Res 2006 & $249 \mathrm{pts}$ & 34 & 211 & $4,00 E-06$ \\
\hline 8 & Sotiriou & JNCI 2006 & $125 \mathrm{pts}$ & 34 & 85 & $4,40 E-06$ \\
\hline 9 & Bild & Nature 2005 & $158 \mathrm{pts}$ & 48 & 110 & $8,20 \mathrm{E}-06$ \\
\hline 10 & Boersma & Int J C ancer 2008 & $95 \mathrm{pts}$ & 52 & 41 & $1,30 \mathrm{E}-05$ \\
\hline 11 & Chin & Cancer Cell 2006 & $118 \mathrm{pts}$ & 43 & 75 & $7,80 \mathrm{E}-05$ \\
\hline 12 & Minn & Nature 2005 & $99 \mathrm{pts}$ & 42 & 57 & $1,60 \mathrm{E}-04$ \\
\hline 13 & Hess & JCO 2006 & $133 \mathrm{pts}$ & 51 & 82 & 0,009 \\
\hline 14 & Perou & Nature 2000 & $20 \mathrm{pts}$ & 9 & 26 & 0,037 \\
\hline 15 & Sorlie & PNAS 2001 & 78 pts & 18 & 56 & 0,063 \\
\hline
\end{tabular}

Total

$\begin{array}{lll}2546 & 713 & 1724\end{array}$

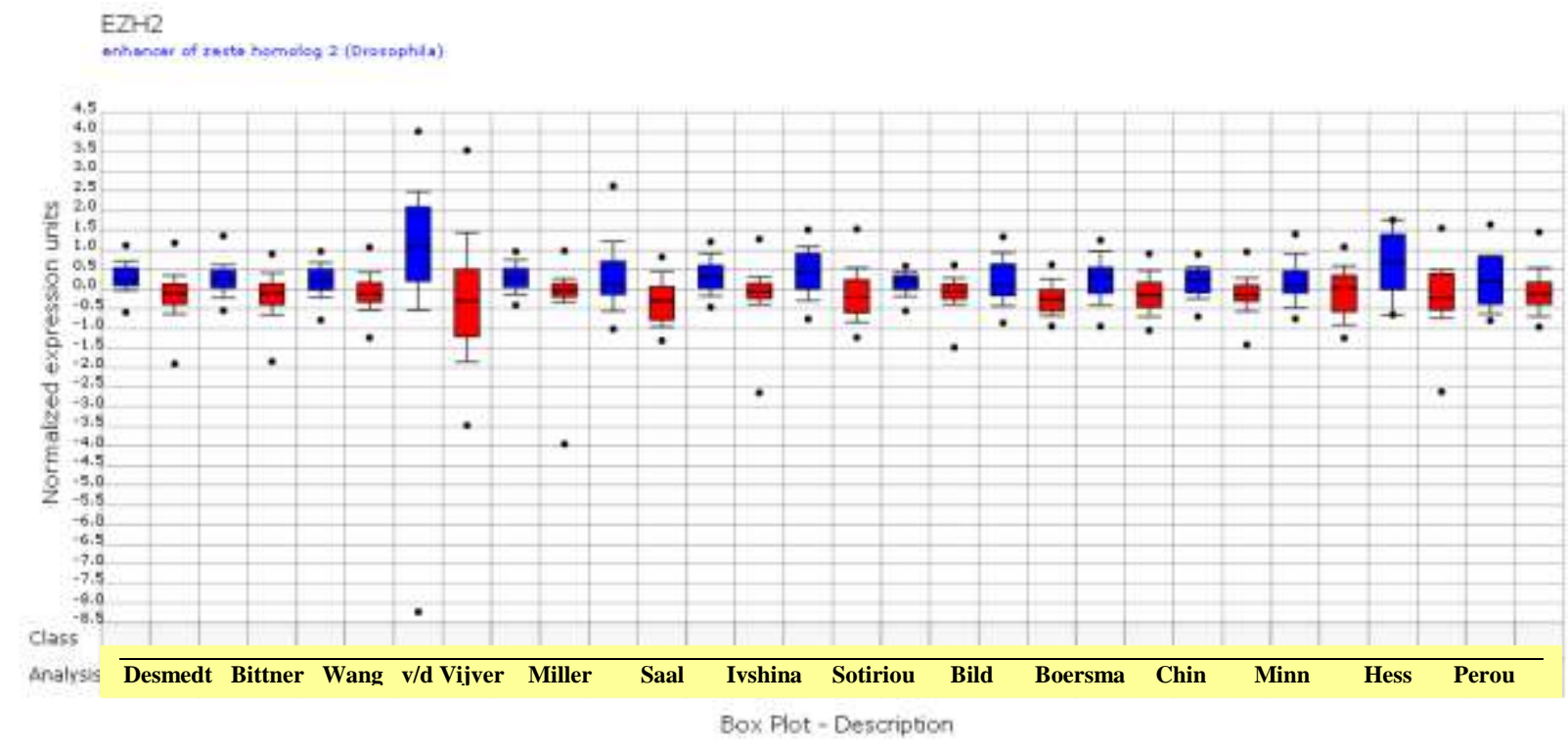


